\title{
HERMITE-HADAMARD'S INEQUALITIES FOR MULTIVARIATE $g$-CONVEX FUNCTIONS
}

\author{
M. KlaričIĆ BAKUlA, E. NeUman, J. PEČARIĆ AND VidA ŠIMIĆ
}

Abstract. Refinements of generalized Hermite-Hadamard's inequalities for multivariate $g$-convex functions are given. Since special instances of the $g$-convex functions include the $r$-convex and the logarithmically convex functions, those inequalities also give refinements of the HermiteHadamard's ineqalities for these families of functions.

Mathematics subject classification (2000): 26D15, 26B25.

Key words and phrases: Hermite-Hadamard's inequalities, $g$-convexity, $r$-convexity, log -convexity, functional Stolarsky means, logarithmic mean.

\section{REFERENCES}

[1] B. C. CARLSON, Special Functions of Applied Mathematics, Academic Press, New York, 1977.

[2] S. S. DRAGOMIR, Refinements of the Hermite-Hadamard integral inequality for log-convex functions, Austral. Math. Gazette 28, (2001), 129-134.

[3] S. S. Dragomir, B. Mond, Integral inequalities of Hadamard's type for log-convex functions, Demonstratio Math. 31, 2 (1998), 354-364.

[4] S. S. DRAGOMIR, C. E. M. PEARCE, Selected Topics on Hermite-Hadamard Inequalities and Applications, RGMIA Monographs, Victoria University, 2000.

(Online: http://rgmia.vu.edu.au/monographs/hermite_hadamard.html).

[5] E. NeUman, Inequalities involving multivariate convex functions II, Proc. Amer. Math. Soc., 109, (1990), 965-974.

[6] E. Neuman,The weighted logarithmic mean, J. Math. Anal. Appl., 188, (1994), 885-900.

[7] E. Neuman, J. SÁNDOR, Inequalities involving Stolarsky and Gini means, Math. Pannonica, 14, (2003), $29-44$.

[8] E. Neuman, C. E. M. Pearce, J. PeČArić And V. Šimić, The generalized Hadamard's inequality, $g$-convexity and functional Stolarsky means, Bull. Austral. Math. Soc.68, (2003), 303-316.

[9] C. E. M. PEARCE, J. PEČARIĆ AND V. ŠIMIĆ, Stolarsky means and Hadamard's inequality, J. Math. Anal. Appl., 220, (1998), 99-109.

[10] C. E. M. PEARCE, J. PEČARIĆ AND V. ŠIMIĆ, Functional Stolarsky Means, Mathematical Inequalities and Applications 2, 4 (1999), 479-489.

[11] J. E. PeČarić, F. Proschan And Y. L. Tong, Convex Functions, Partial Orderings and Statistical Applications, Academic Press, Boston, 1992.

[12] J. PeČARIĆ, V. ŠIMIĆ, Stolarsky-Tobey mean in $n$ variables, Mathematical Inequalities and Applications 2, 3 (1999), 325-341. 